This item was submitted to Loughborough's Research Repository by the author.

Items in Figshare are protected by copyright, with all rights reserved, unless otherwise indicated.

\title{
Three-dimensional analysis of the effect of material randomness on the damage behaviour of CFRP laminates with stochastic cohesive-zone elements
}

\section{PLEASE CITE THE PUBLISHED VERSION}

http://dx.doi.org/10.1007/s10443-013-9354-3

\section{PUBLISHER}

(C) Springer Science+Business Media

\section{VERSION}

AM (Accepted Manuscript)

\section{LICENCE}

CC BY-NC-ND 4.0

\section{REPOSITORY RECORD}

Khokhar, Zahid R., lan A. Ashcroft, and Vadim V. Silberschmidt. 2019. "Three-dimensional Analysis of the Effect of Material Randomness on the Damage Behaviour of CFRP Laminates with Stochastic Cohesive-zone Elements". figshare. https://hdl.handle.net/2134/14536. 
This item was submitted to Loughborough's Institutional Repository (https://dspace.lboro.ac.uk/) by the author and is made available under the following Creative Commons Licence conditions.

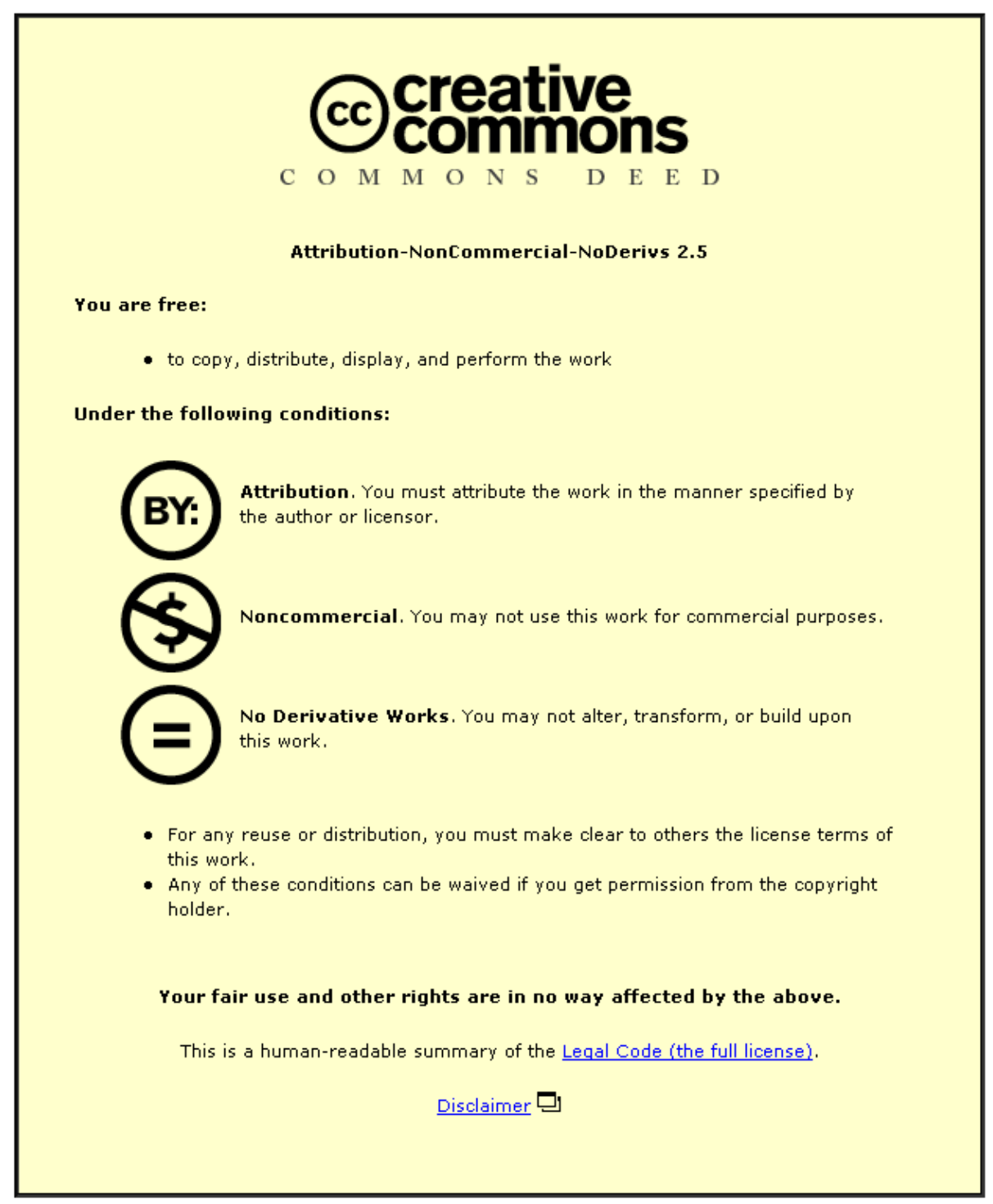

For the full text of this licence, please go to: http://creativecommons.org/licenses/by-nc-nd/2.5/ 


\title{
Three-dimensional analysis of the effect of material randomness on the damage behaviour of CFRP laminates with stochastic cohesive-zone elements
}

\author{
Zahid R. Khokhar ${ }^{1}$, lan A. Ashcroft ${ }^{2 *}$ and Vadim V. Silberschmidt ${ }^{1}$ \\ ${ }^{1}$ Wolfson School of Mechanical and Manufacturing Engineering, Loughborough University, \\ Loughborough, Leicestershire, LE11 3TU, UK \\ ${ }^{2}$ Faculty of Engineering, University of Nottingham, University Park, Nottingham, NG7 2RD
}

\begin{abstract}
Laminated carbon fibre-reinforced polymer (CFRP) composites are already well established in structural applications where high specific strength and stiffness are required. Damage in these laminates is usually localised and may involve numerous mechanisms, such as matrix cracking, laminate delamination, fibre de-bonding or fibre breakage. Microstructures in CFRPs are nonuniform and irregular, resulting in an element of randomness in the localised damage. This may in turn affect the global properties and failure parameters of components made of CFRPs. This raises the question of whether the inherent stochasticity of localised damage is of significance in terms of the global properties and design methods for such materials.

This paper presents the numerical modelling based analysis of the effect of material randomness on delamination damage in CFRP materials by the implementation of stochastic cohesive-zone model (CZM) within the framework of the finite-element (FE) method. The initiation and propagation of delamination in a unidirectional CFRP double-cantilever beam (DCB) specimen loaded under mode-I was analyzed, accounting for the inherent microstructural stochasticity exhibited by such laminates via the stochastic CZM. Various statistical realizations for a half-scatter of $50 \%$ of fracture energy were performed, with probability a distribution based on Weibull's two-parameter probability density function. The damaged area and the crack lengths in laminates were analyzed, and the results showed higher values of those parameters for random realizations compared to the uniform case for the same levels of applied displacement. This indicates that deterministic analysis of composites using average properties may be non-conservative and a method based on probability may be more appropriate.
\end{abstract}

Keywords: CFRP laminates; delamination; stochasticity; cohesive zone elements; microstructural randomness; Weibull's distribution. 


\section{Introduction}

An important factor affecting the localised damage evolution in carbon fibre-reinforced laminates is the randomness exhibited by these laminates at the microstructure level. In most of the analyses of fibre-reinforced composite materials, they are treated in a deterministic way i.e., the material has (or is implicitly assumed to have) an ordered (deterministic) distribution of fibres. However, in reality, the microstructure of fibre- reinforced composites is far from ordered as there is significant variability in the distribution of the fibres in the matrix. The manufacturing processes of fibre-reinforced laminates lead to macroscopic heterogeneity of the resultant materials. As a result of this non-uniformity, variations in the local properties of these laminates arise, which affect the localised processes of damage initiation and evolution. Baxevanakis et al. [1] demonstrated a high level of spatial non-uniformity in a composite, using an image analysis technique to study fibre distributions in cross-sectional areas of T300/914 specimens. The effect of such microstructural randomness on the localised failure mechanisms was demonstrated by Silberschmidt [2], who demonstrated that cross-ply carbon-epoxy laminates exhibit a considerable extent of randomness in the distribution of transverse cracks and discussed the effect of microstructural randomness on the distribution of matrix cracks in hese laminates [3]. It was concluded that the behaviour of specimens with multiple matrix cracks cannot be always reduced to the analysis of a single traditional unit cell as an area limited by two neighbouring cracks under the assumption of equal crack spacing. In agreement with this observation, Trias et al. [4] showed from a comparison of stress and strain distributions obtained with periodic and random models of a carbon fibre-reinforced polymer, that periodic models can be used to assess effective properties but random ones must be considered for the simulation of local phenomena such as damage accumulation or matrix cracking. A further contribution by Silberschmidt [5] presented a lattice model which was used to study damage and fracture evolution in laminates, linking microstructural randomness with macroscopic properties and demonstrating that a random character in the fibres' distribution results in fluctuations of local elastic moduli, the bounds of which depend on the characteristic length scale. Yanga et al. [6] simulated two-dimensional crack propagation in quasi-brittle materials considering both uniform and randomly variable fracture properties. They concluded that the use of the homogeneous model leads to erroneous predictions of crack patterns and load-displacement curves whereas the models where the variable fracture properties were taken into account, presented a more realistic representation. Khokhar et al. [7] studied the effect of microstructural randomness in a cross-ply laminate beam under bending with the introduction of matrix cracks with varying spacings and delamination zones. A considerable 
difference in the values of reduction in stiffness between cases with different crack spacings suggested that the assumption of averaged distributions of defects can lead to unreliable predictions of structural response. In another work by Khokhar et al. [8], based on twodimensional DCB models, it was concluded that the variation in the fracture properties undertaken to analyze the effect of material randomness using stochastic cohesive zone elements had a potentially significant effect on the predictions of the performance of CFRP laminates.

The results from the studies described above work provide a case that the material randomness observed in laminates needs to be accounted for when designing with these materials, particularly when designing against failure. In addition, the previous literature in this area indicates the need for a more detailed analysis based on three-dimensional simulations. In the previous two-dimensional analysis [8], the variation of fracture parameters within the cohesive layer was only one-dimensional, i.e., only along the length of the double-cantilever beam. To have a more complete picture of the effect of material randomness on the specimen under study, a three-dimensional analysis is necessary in order to investigate the effect of twodimensional variations in the microstructure in the plane of delamination. This is supported by a previous study that showed that fibres are aligned in their longitudinal direction in most unidirectional laminates but if we look at the transverse cross-sections the arrangements of these fibres in the matrix are usually found to be random [9].

Recently, the authors [10] presented their initial investigations using three-dimensional analyses where the effects of a statistical variation of the critical load for a DCB were reported. This work indicates that scatter in the results from testing CFRPs is an inevitable consequence of the microstructural randomness and that this can be investigated through the use of stochastic cohesive zone elements. It was also been shown that the median value of results from models with stochastic variation in properties does not necessarily coincide with the results from using uniform, mean properties. The current study will undertake the threedimensional analysis in more detail and statistical analysis of various output parameters will be presented in addition to the critical load. 
The term 'uniform model' is used in this paper for models that are implemented using uniform fracture properties throughout the cohesive layer. For all the models, where simulations were based on variations of fracture parameters, the models will be referred to as 'random model'.

Initially, the results from a uniform model will be presented, where there is no variation in the fracture energy. Following this, the results from the random models are presented, where the variations in the fracture energy are introduced. Three models are used to analyse the shape of crack fronts, track the delamination crack paths, analyse the variation in damage within the cohesive layer in the plane of delamination, and determine the critical loads and delamination crack lengths. The output statistics of parameters from various statistical realizations will be presented, using spatially random levels of fracture energy in the cohesive-zone model, and the results from random model will be compared with those for the uniform model.

\section{Finite Element Analysis of DCB}

Three dimensional finite element analysis (FEA) of a double cantilever beam (DCB) with unidirectional composite adherends was carried out using the commercial software MSC Marc, the boundary conditions of the model being shown in Fig. 1. The specimen was $125 \mathrm{~mm}$ long and $20 \mathrm{~mm}$ wide with twenty four $0.125 \mathrm{~mm}$ thick plies giving a total thickness of $3 \mathrm{~mm}$. An initial crack length of $35 \mathrm{~mm}$ was introduced into the model.

The element edge length used for the mesh was $0.15 \mathrm{~mm}$ and the total number of elements for this model was 77,300 (including 6010 cohesive elements). Owing to symmetry, half of the DCB model, could be analyzed for the deterministic (uniform) case but as we later introduce material randomness in the model a full model was used for consistency. The element used for the cantilever arms was MSC Marc Type 7, which is an eight-node, iso-parametric, arbitrary hexahedral element. The cohesive zone element (CZE) used was Type 188. The mechanical properties of the unidirectional CFRP are shown in Table 1. The specimen was $125 \mathrm{~mm}$ long and $20 \mathrm{~mm}$ wide with twenty four $0.125 \mathrm{~mm}$ thick plies giving a total thickness of $3 \mathrm{~mm}$. An initial crack length of $35 \mathrm{~mm}$ was introduced into the model. 
Displacements

constrained in all three

directions, $x, y$, and $z$

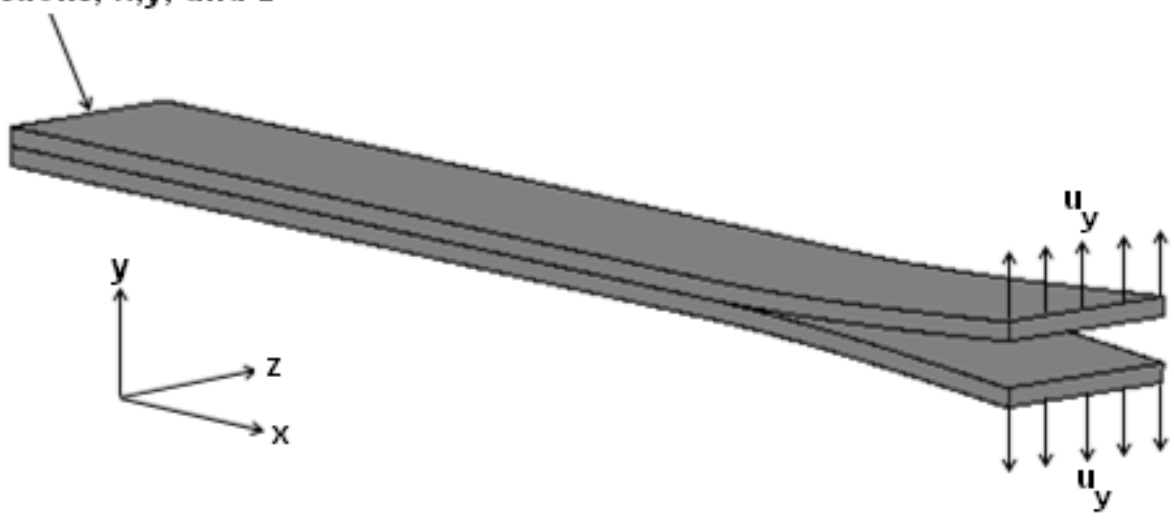

Fig. $13 \mathrm{D}$ model of double-cantilever beam

The cohesive elements were defined using a bilinear traction-separation law, which is described in more detail in [10]. The material properties used in the model are summarized in Table 1.

\begin{tabular}{lc}
\hline \multicolumn{1}{c}{ Parameter } & Value \\
\hline \multicolumn{2}{c}{ CFRP Properties } \\
\hline$E_{11}=137 \mathrm{GPa}$ \\
$E_{22}, E_{33}=8 \mathrm{GPa}$ \\
$G_{12}, G_{13}=4 \mathrm{GPa}$ \\
$G_{32}=3.2 \mathrm{GPa}$ \\
\hline Elastic moduli & $v_{12}, v_{13}=0.31$ \\
& $v_{23}=0.52$ \\
\hline Coisson's ratios & \\
\hline Fracture energy, $G_{\mathrm{F}}$ & $0.257 \mathrm{~kJ} / \mathrm{m}^{2}$ \\
\hline Tripping traction, $\sigma_{\text {max }}$ & $50 \mathrm{MPa}$ \\
\hline & $1.6 \times 10^{6} \mathrm{~N} / \mathrm{mm}^{3}$ \\
\hline
\end{tabular}


Table 1 Material parameters used in uniform model

\section{Implementation of material variability in cohesive zone model}

The inherent material stochasticity exhibited by CFRP laminates was introduced by spatially varying the fracture energy in the CZE based on a two-parameter Weibull's distribution function. This was achieved by first representing the fracture energy of cohesive elements by a distribution with a constant probability density for a defined scatter width $\left[G_{\mathrm{F}}{ }^{{ }^{i n}}, G_{\mathrm{F}}{ }^{\max }\right]$; in this case with a set of magnitudes between the two extreme values $\left(0.5 G_{\mathrm{F}}\right.$ and $\left.1.5 G_{\mathrm{F}}\right)$ was produced using a random number generator. The choice of the bounds for fracture energy for this composite is defined by the extent of fluctuations of its local volume fraction of fibres at the given length scale (see more in [5]). The random numbers generated were then transformed to comply with the Weibull's distribution, producing a set of random fracture energies based on that distribution. The two-parameter Weibull probability density function for a random variable $G_{\mathrm{F}}$ is given by

$$
P\left(G_{\mathrm{F}}\right)=\frac{\alpha}{\beta}\left(\frac{G_{\mathrm{F}}}{\beta}\right)^{\alpha-1} \exp \left(-\left(\frac{G_{\mathrm{F}}}{\beta}\right)^{\alpha}\right)
$$

where $\alpha>0$ is the shape parameter and $\beta>0$ is the scale parameter of the distribution. The corresponding cumulative distribution function is given by the function

$$
C\left(G_{\mathrm{F}}\right)=1-\exp \left(-\left(\frac{G_{\mathrm{F}}}{\beta}\right)^{\alpha}\right) .
$$

A choice of the Weibull's parameters, in general case, should be based on statistics of the experimental fracture data for the length scale of interest. The lack of this information resulted in a selection of the probability distribution function that covers $\left[G_{\mathrm{F}}{ }^{\min }, G_{\mathrm{F}}{ }^{\max }\right]$, with its peak coinsiding with $G_{\mathrm{F}}$ (thus defining 6 ) and a moderate decline towards the ends of this interval. The same parameters were retained in all the simulations for comparability. The number of data points $N$ in the set was equal to the number of nodes in the cohesive layer of the finite- 
element model, to which it was assigned. The same average magnitude of the fracture energy, given by

$$
\left\langle G_{\mathrm{F}}\right\rangle=\frac{1}{N} \sum_{i=1}^{N} G_{\mathrm{F} i}
$$

which equal to the fracture energy used in the uniform model (i.e. $G_{\mathrm{F}}=0.257 \mathrm{~kJ} / \mathrm{m}^{2}$ ), was used in each statistical realisation. Any small deviations arising from this average magnitude due to the discretization of the Weibull's distribution function were removed by a renormalization based on $\left\langle G_{\mathrm{F}}\right\rangle$. A number of statistical realizations were studied using this method where random numbers were generated with different seeds. A similar distribution has been used by other researchers to introduce spatially non-uniform fracture energies in non-linear fracture mechanics problems, one of them being the work by Yang and Frank Xu [11].

The fracture energy scatter was introduced with a view to investigate the effect of varying the fracture energy (mode-I fracture energy in this case) on delamination initiation and propagation in a DCB specimen. It can be seen from the Fig. 2 that for the same values of the opening displacements, by changing the value of fracture energy from $G_{\mathrm{F}}$ to $0.5 G_{\mathrm{F}}$, the initial stiffness of the cohesive law, and the tripping traction decreases (Fig. 2). Varying fracture energy from $G_{\mathrm{F}}$ to $1.5 G_{\mathrm{F}}$ leads to an increase in the values of both the tripping traction and initial stiffness, keeping the values of both the displacements constant.

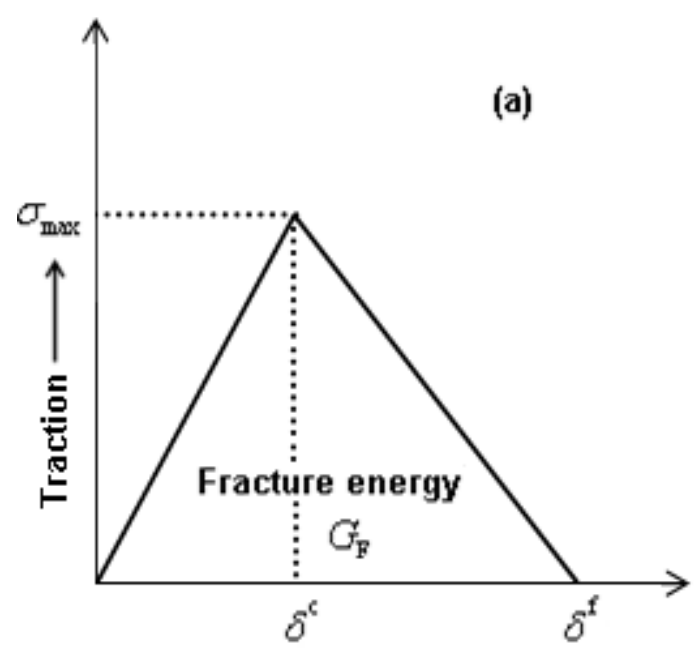

Opening displacement $\delta$

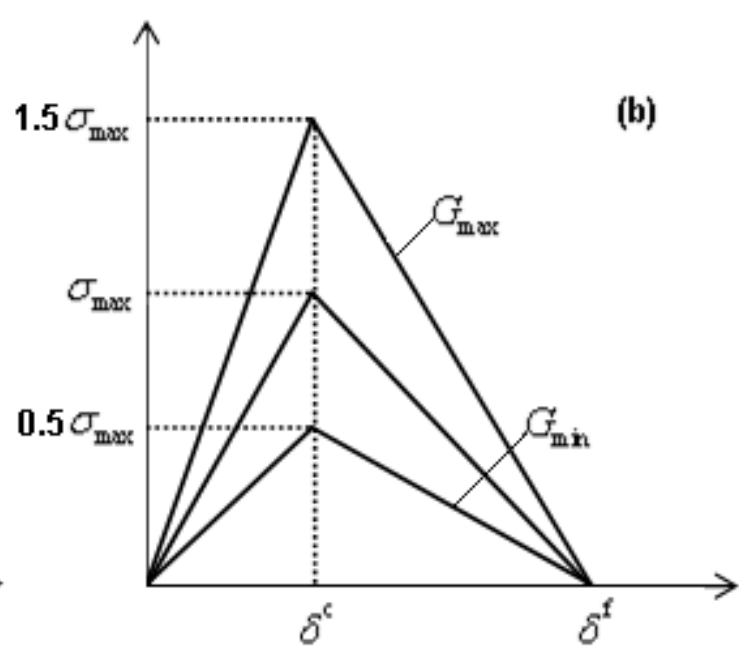

Opening displacement $\delta$

Fig. 2 Bilinear traction-separation law: (a) standard formulation; (b) bounds for fracture energy (random formulation) 


\section{Analysis of results}

Analysis of the results was based on comparison of a number of different parameters obtained in the numerical simulations. These included:

- Strain ahead of the crack tip at delamination initiation;

- Effect of crack propagation on stress distribution;

- Shape of crack fronts;

O Crack lengths;

- Damaged area and its variation across the width of the $D C B$;

- Critical loads and displacements

Detailed analyses of the output statistics for all these parameters are presented in the form of probability density functions (PDFs) and cumulative distribution functions (CDFs). The probability density function describes the relative probability of a random variable to occur at a given point, in the area focused for the observation. The cumulative distribution function (CDF) completely describes the probability distribution of a real-valued random variable. These analyses are described in the following sections.

\subsection{Analysis of strain at delamination initiation}

Strain in the loading direction, i.e. $\varepsilon_{22}$, ahead of the crack tip was analysed, as shown in Fig. 3, where the DCB model and the enlarged part of the specimen ahead of crack tip are shown with the strain contours. The value of strain $\left(\varepsilon_{22}\right)$ at delamination initiation was defined by complete failure of the first element in the cohesive layer. The output statistics of this parameter are shown in the probability plot in Fig. 4 . The plot demonstrates that the values of strain at delamination initiation vary between $0.35 \%$ and $0.45 \%$ in the various realizations of the random model, with the majority of the values concentrated in the upper half of these bounds (i.e. between $0.4 \%$ and $0.45 \%$ ). This compares with a value of $0.37 \%$ in the uniform model. 


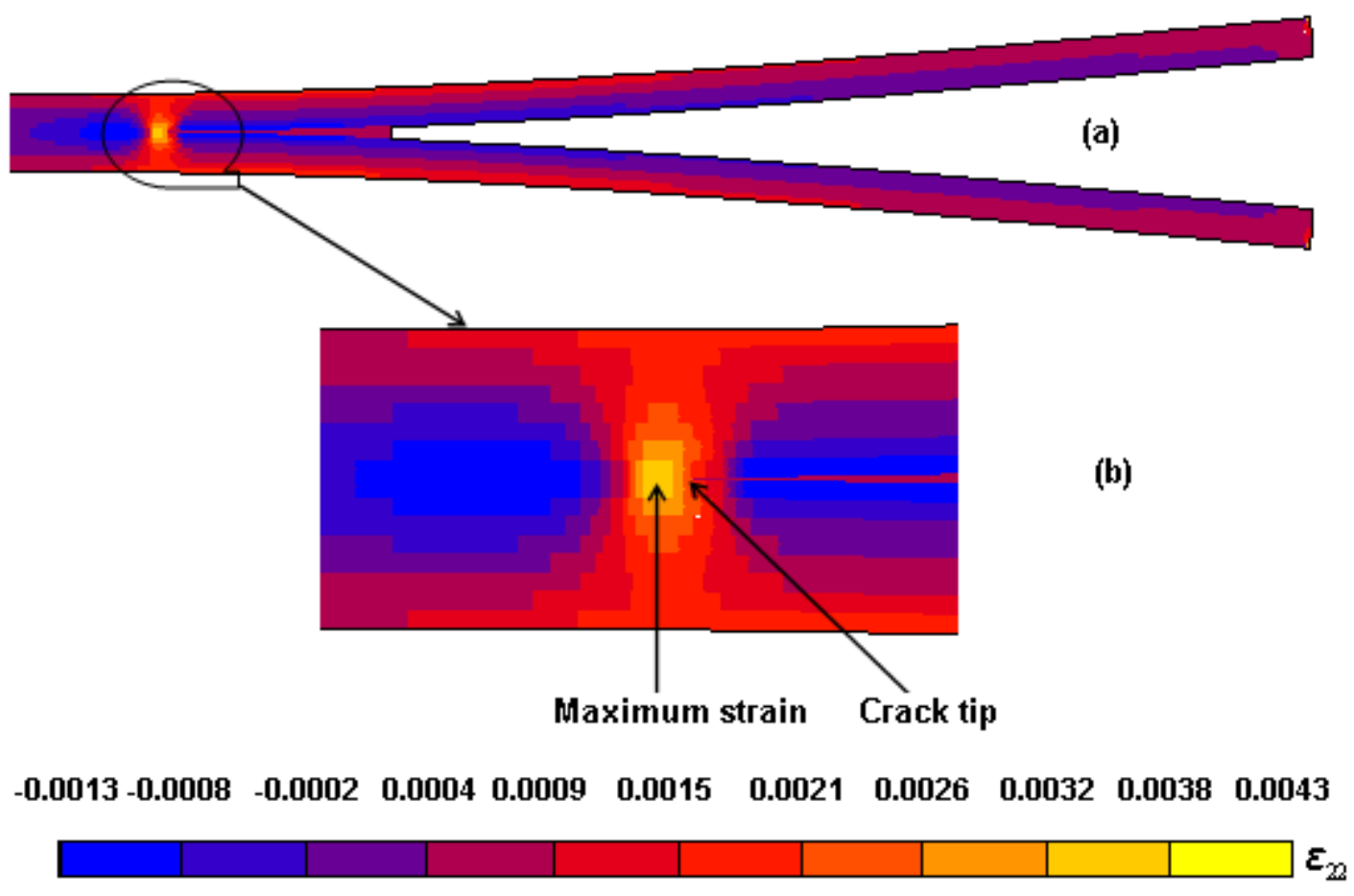

Fig. 3 Location for value of strain $\left(\varepsilon_{22}\right)$ at delamination crack initiation: (a) deformed DCB model; (b) zoomed area ahead of crack tip

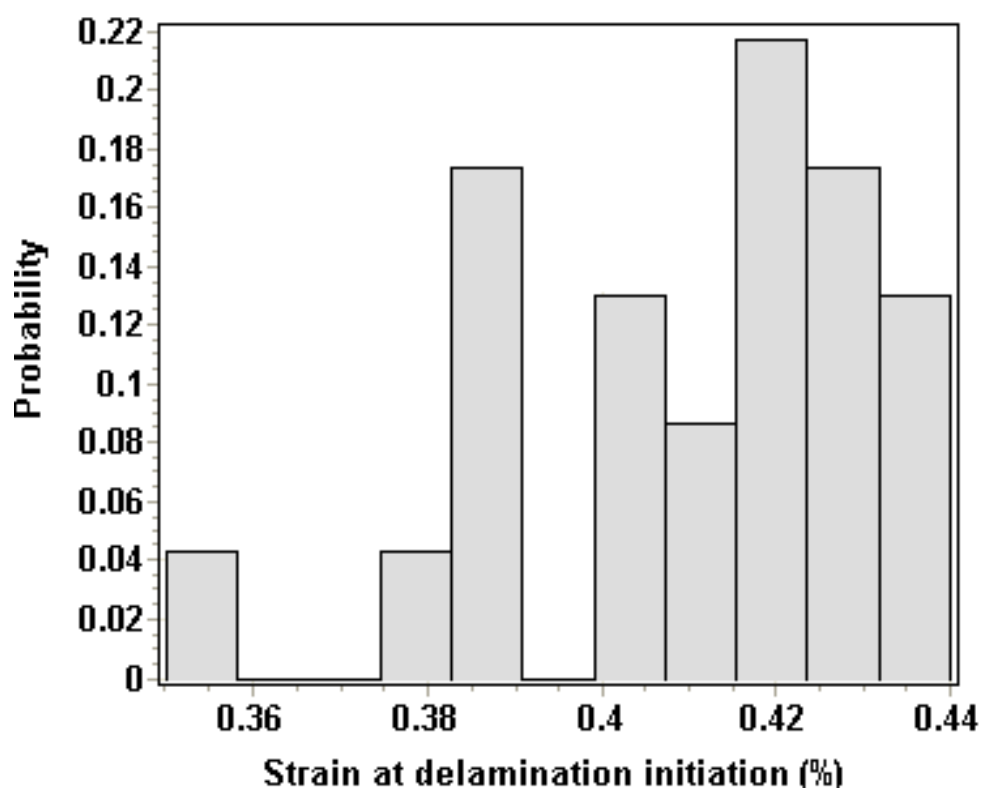

Fig. 4 Probability density plot for strain $\left(\varepsilon_{22}\right)$ at delamination initiation

The probability density function for the strain at delamination initiation is shown in Fig. 5 (a). The value obtained from the uniform model $(0.37 \%)$ and the mean $(0.41 \%)$ of the data obtained from the random statistical realizations are also indicated on the figure and it can be seen that there is a shift in the mean value between the uniform and random models. The PDF is negatively-skewed (skewed left), as there are relatively less low values, concentrating the mass of the distribution to the right. The cumulative distribution function (CDF) for the strain at 
delamination initiation is shown in Fig. 5 (b). This function shows that only around $10 \%$ of the values obtained from the random statistical realizations were less than that obtained for the uniform model. The descriptive statistics of the distribution for strain are presented in Table 2, where percentile ranks are also shown. These results clearly show that using the mean value of a parameter doesn't necessarily result in a mean output when localised damage phenomena are involved.

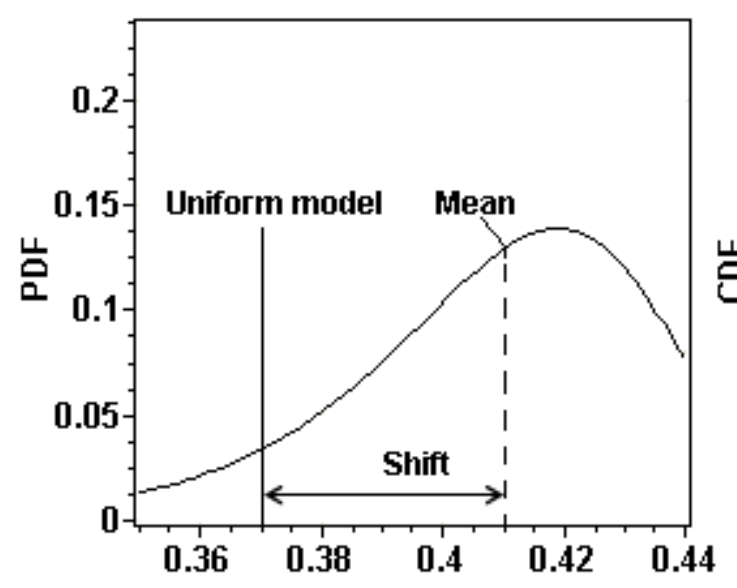

Strain at delamination initiation (\%)

(a)

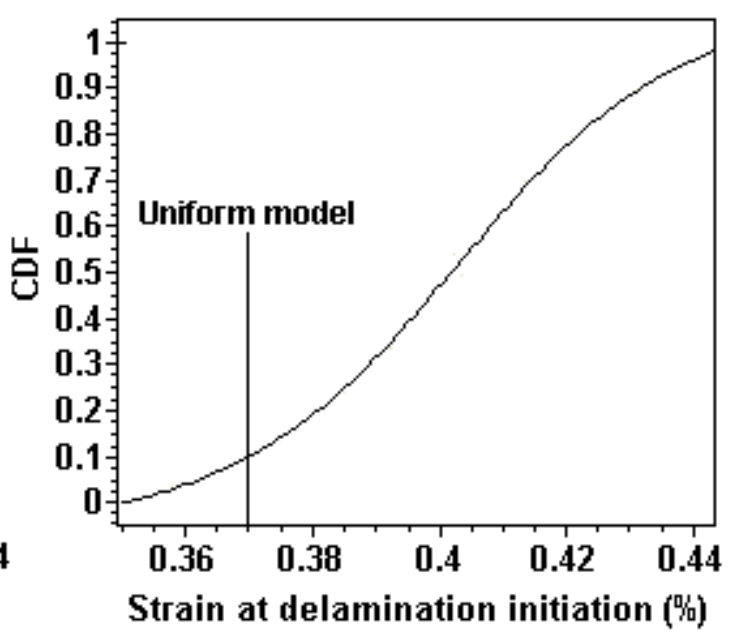

(b)

Fig. 5 Probability density function (PDF) (a) and cumulative distribution function (CDF) (b) for strain level at delamination initiation ( $\alpha=19.4$ and $\beta=0.42)$

Table 2 Descriptive statistics for strain at delamination initiation

\begin{tabular}{|l|c|c|c|c|c|c|c|}
\hline Percentile & Min & $10 \%$ & $\begin{array}{c}25 \% \\
\text { (Q1) }\end{array}$ & $\begin{array}{c}50 \% \\
\text { (Median) }\end{array}$ & $\begin{array}{c}75 \% \\
(\mathrm{Q} 3)\end{array}$ & $90 \%$ & Max \\
\hline Value & 0.35 & 0.384 & 0.39 & 0.42 & 0.43 & 0.44 & 0.44 \\
\hline
\end{tabular}

\subsection{Analysis of stresses}

The critical stress component in the case of the DCB is the stress in the direction of application of displacement i.e. $\sigma_{22}$. In order to highlight the effect of material randomness on the stress distribution, a contour plot of $\sigma_{22}$ is shown in Fig. 6 for one of the random statistical realizations. The stresses ahead of the crack front can be observed in the magnified plot in Fig. 
6 (b), to exhibit non-symmetric patterns about the centre-line of the $D C B$, unlike those observed in the uniform properties model.

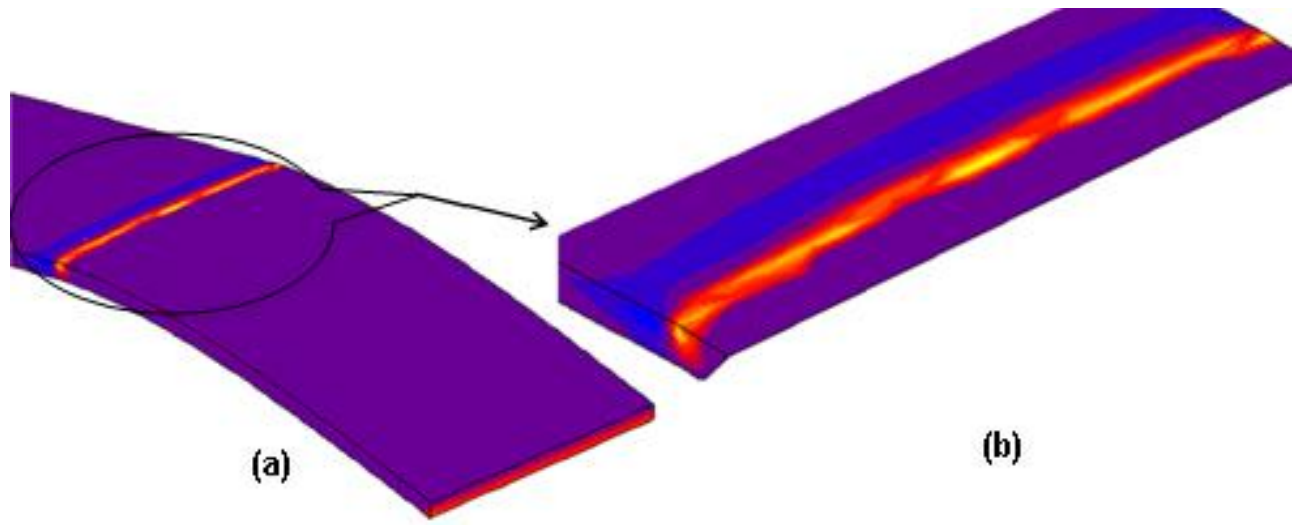

Fig. 6 Contour plot of stress $\sigma_{22}$ for a random model (a) and area magnified ahead of crack

$$
\text { front (b) ( } \left.\varepsilon_{22}=0.004\right)
$$

Contour plots of stress $\sigma_{22}$ for the uniform model for strains ranging from $0.4 \%$ to $0.6 \%$ are shown in Fig. 7 (the view being a plan view of the delamination plane). It can be seen that the stress distribution and crack front are both symmetric about a line through the centre of the sample width. The areas of maximum stress are indicated by the star symbols (-) and the crack propagation can be seen to follow these areas.
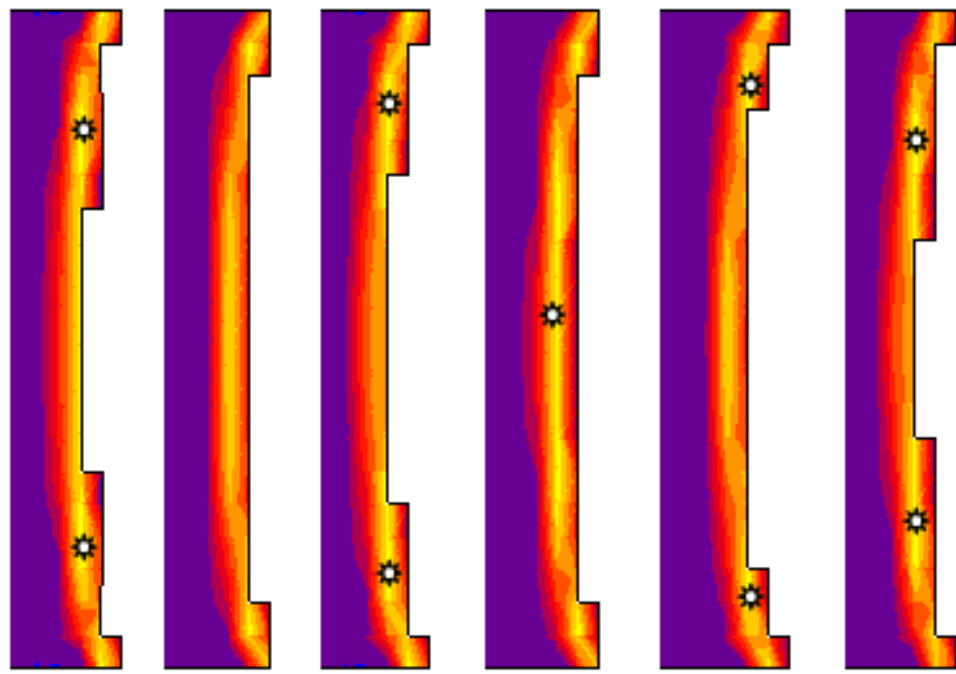

\section{Delamination propagation direction}

Fig. 7 Plots of stress $\sigma_{22}$ for increasing values of applied strain ( $\varepsilon_{22}=0.4 \%$ to $0.6 \%$ ) - uniform model. Areas of maximum stress indicated by 
Contour plots of $\sigma_{22}$ for the one of the random realizations are shown in Fig. 8. As with the uniform model, the potential areas for crack propagation in the random model can be readily identified with the areas of high stress (shown by symbol on Fig. 8), however, in this case the stress distribution and crack front are not symmetric.
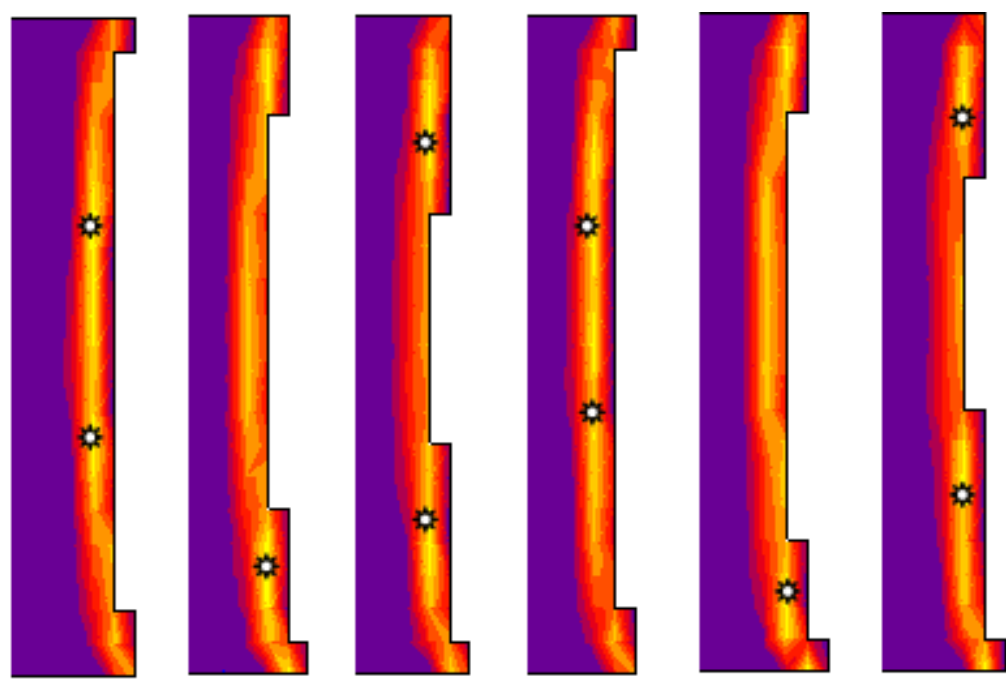

Delamination propagation direction

Fig. 8 Plots of stress $\sigma_{22}$ for different values of applied strain ( $\varepsilon_{22}=0.4 \%$ to $0.6 \%$ ) - random model. Areas of maximum stress indicated by

\subsection{Analysis of crack fronts}

A contour plot of damage in the cohesive-zone layer after crack initiation is shown in Fig. 9, for one of the random cases studied. The non-symmetric nature of the crack front and the propagating process zone is clearly visible, and can be contrasted with the symmetric behaviour seen in the uniform model. Also, fast and comparably slow propagations of delamination ahead of the crack front can be seen in Fig. 9. Plan views of the crack fronts obtained for different statistical realizations (i.e. different spatial distribution of fracture energy for the same Weibull's parameters) are shown in Fig. 10. One of these cases demonstrates a symmetric crack, as shown in Fig. 10 (b). During the course of crack propagation, it was observed that the crack fronts in the random models changed between symmetric and nonsymmetric shapes. After every four to five non-symmetric propagations, the crack became symmetric and then returned to non-symmetric behaviour. This transition between symmetric 
and non-symmetric configurations can be observed in the 2D plot of propagation of delamination with increasing displacement in Fig. 11 for one of the random realizations.

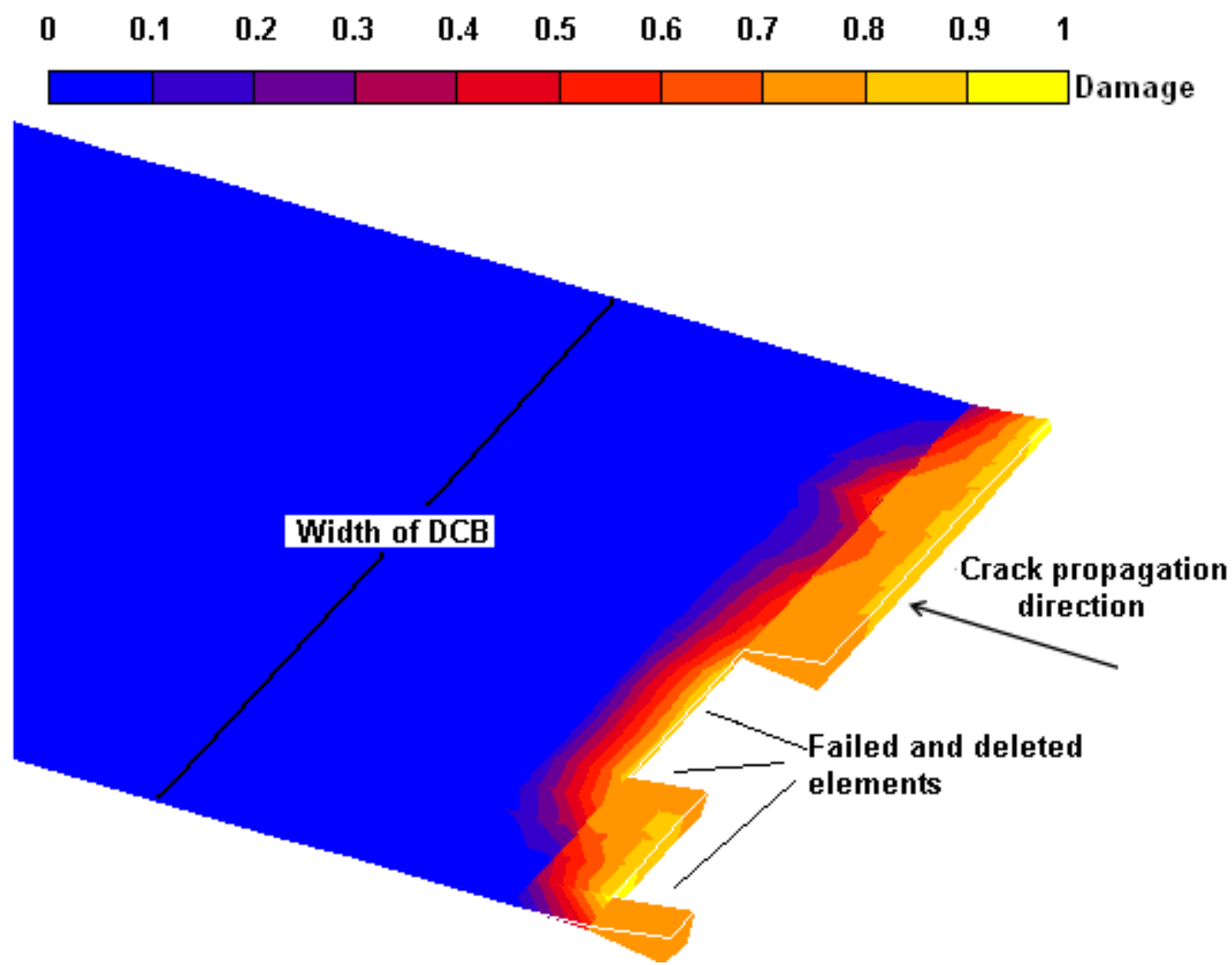

Fig. 9 Delamination crack propagation in random model $\left(\varepsilon_{22}=0.004\right)$
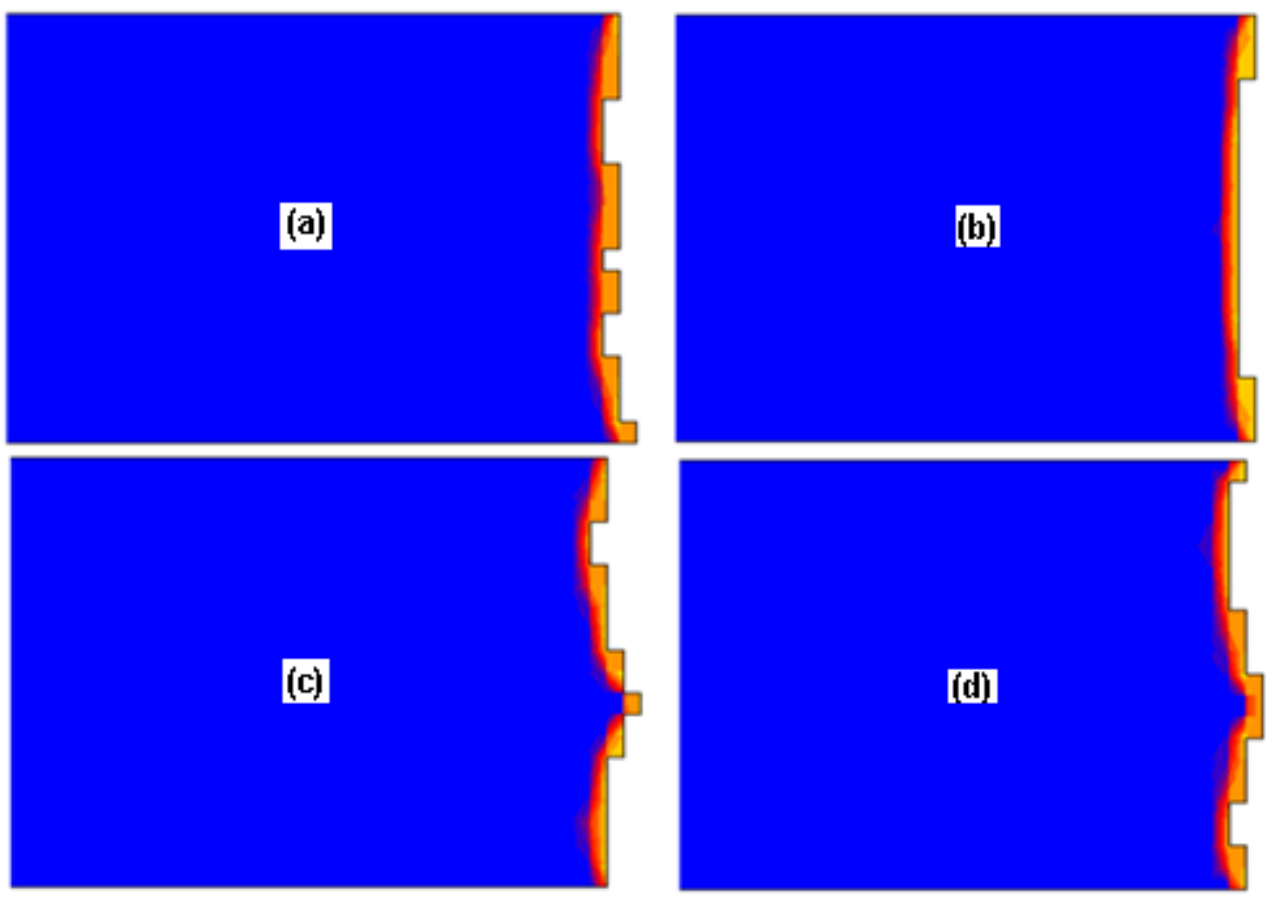

Fig. 10 Delamination propagation for same macroscopic deformation $\left(\varepsilon_{22}=0.004\right)$ for four different statistical realizations 


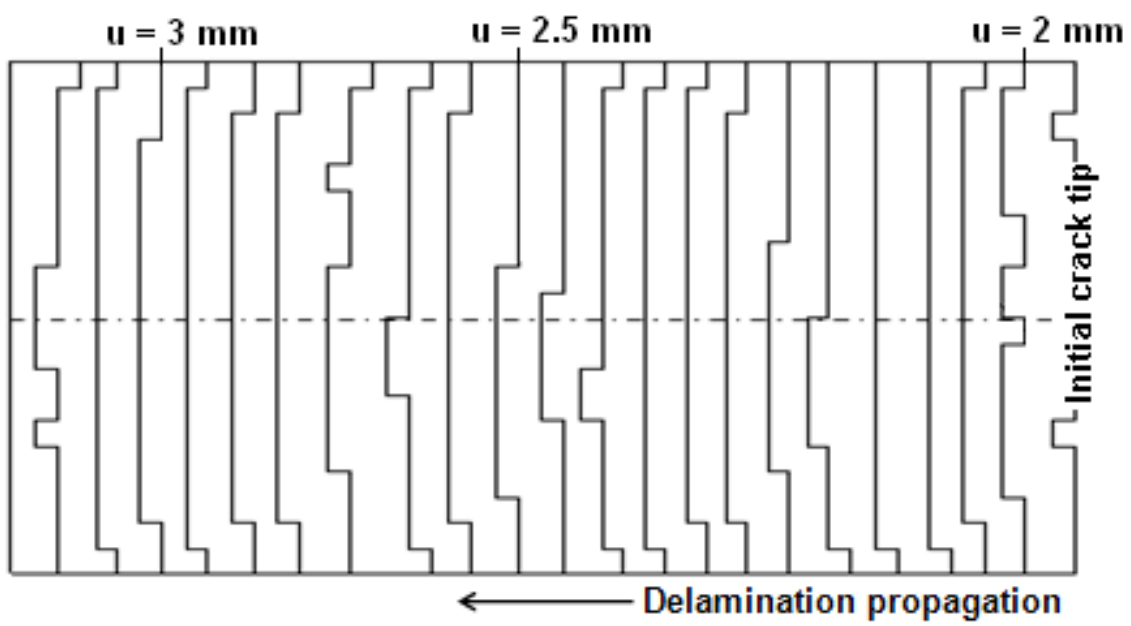

Fig. 11 Evolution of crack front shape for one statistical realization

\subsection{Analysis of crack lengths}

Three different crack length parameters, namely minimum (Min), mean (Mean) and maximum (Max) crack length were investigated, as defined in Fig. 12. The results from some of the various statistical realizations of the random model are compared with the uniform model in Fig. 13, Fig. 14 and Fig. 15. It can be seen in Fig. 13 that the crack length in the random models is initially lower than that in the uniform model, however, after approximately $2.3 \mathrm{~mm}$ applied displacement, a faster crack growth was experienced by the random models compared to the uniform model, and hence, higher values of crack length were observed compared to the uniform model. It is notable that although there are differences between the behaviour of the various random models, they all show this same trend with respect to the uniform model. Similar trends were also seen with the mean and maximum crack lengths, as seen in Figs. 14 and 15. Another feature that can be seen in the Figure 13 is that the crack development for uniform as well as for random models showed a stepped propagation pattern, which is a feature of the element based CZM used. However this is less evident for the mean crack length for the uniform model at low applied displacements, which can be attributed to the stable and progressive, symmetric crack growth, as shown in Fig. 7, and the averaging effect of taking the mean crack length. 


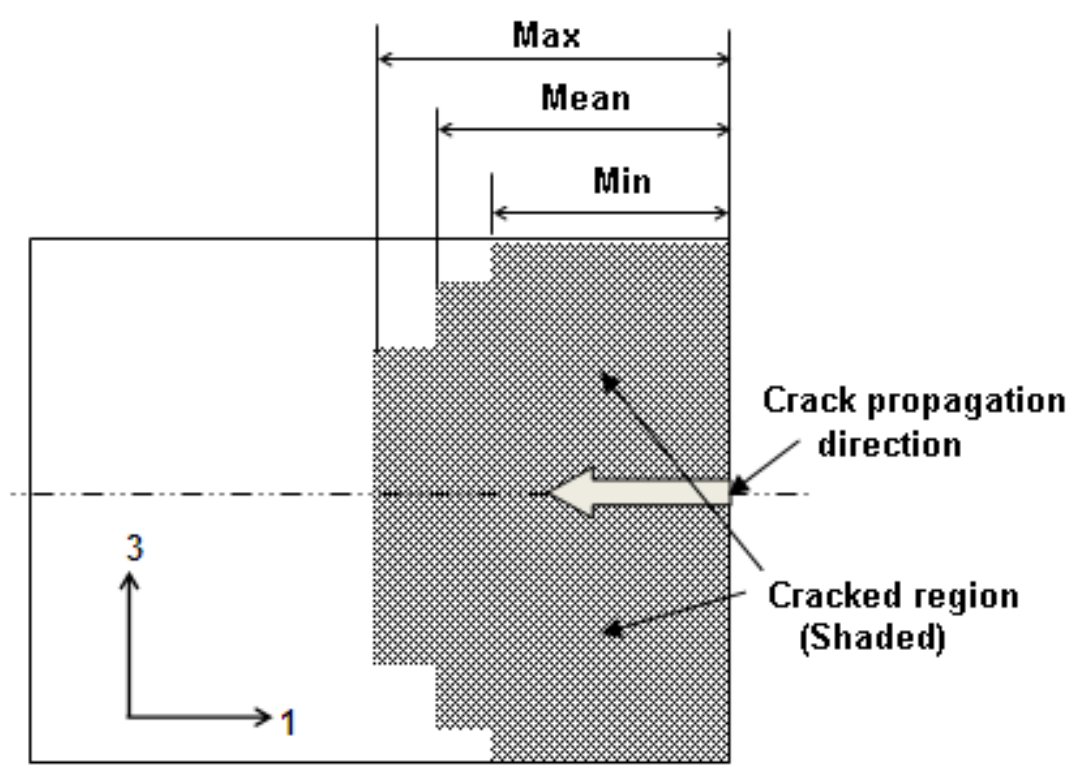

Fig. 12 Different crack lengths analysed

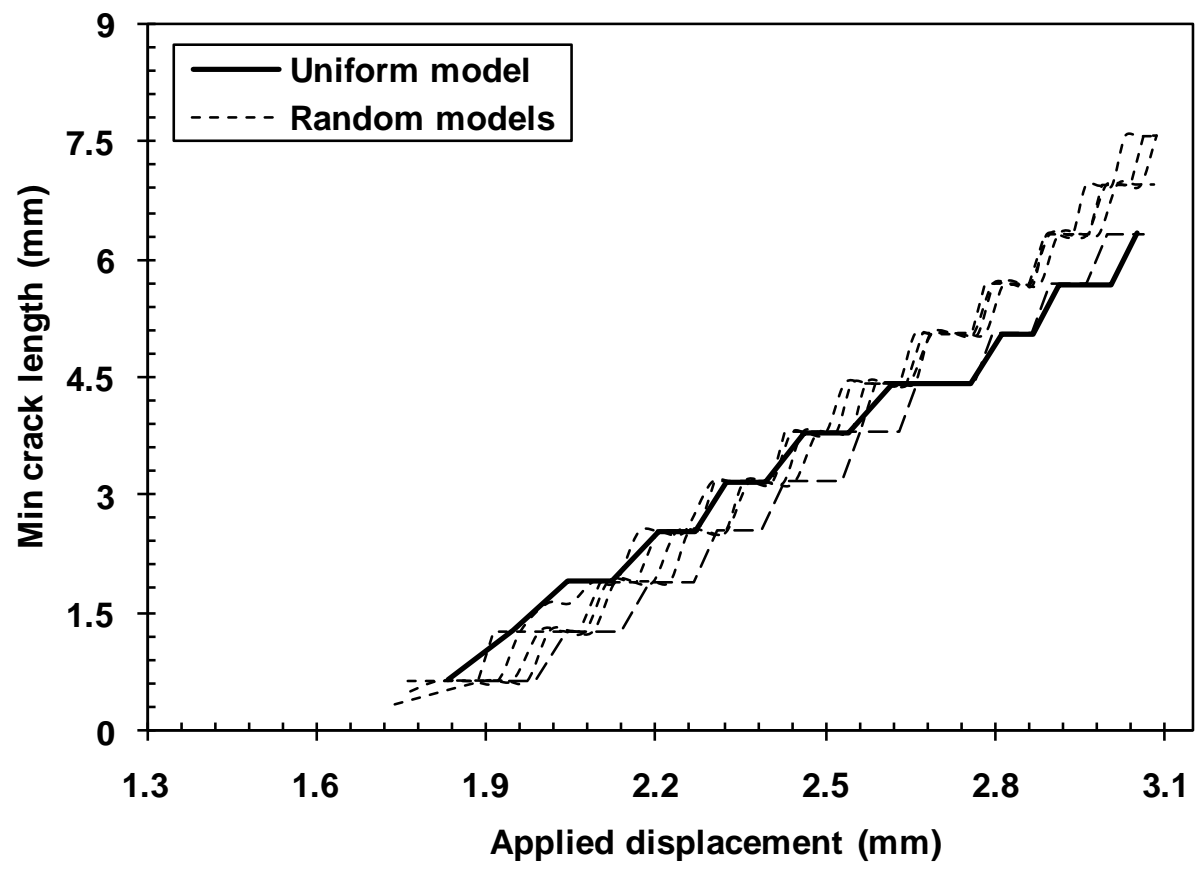

Fig. 13 Min crack length as a function of applied displacement 


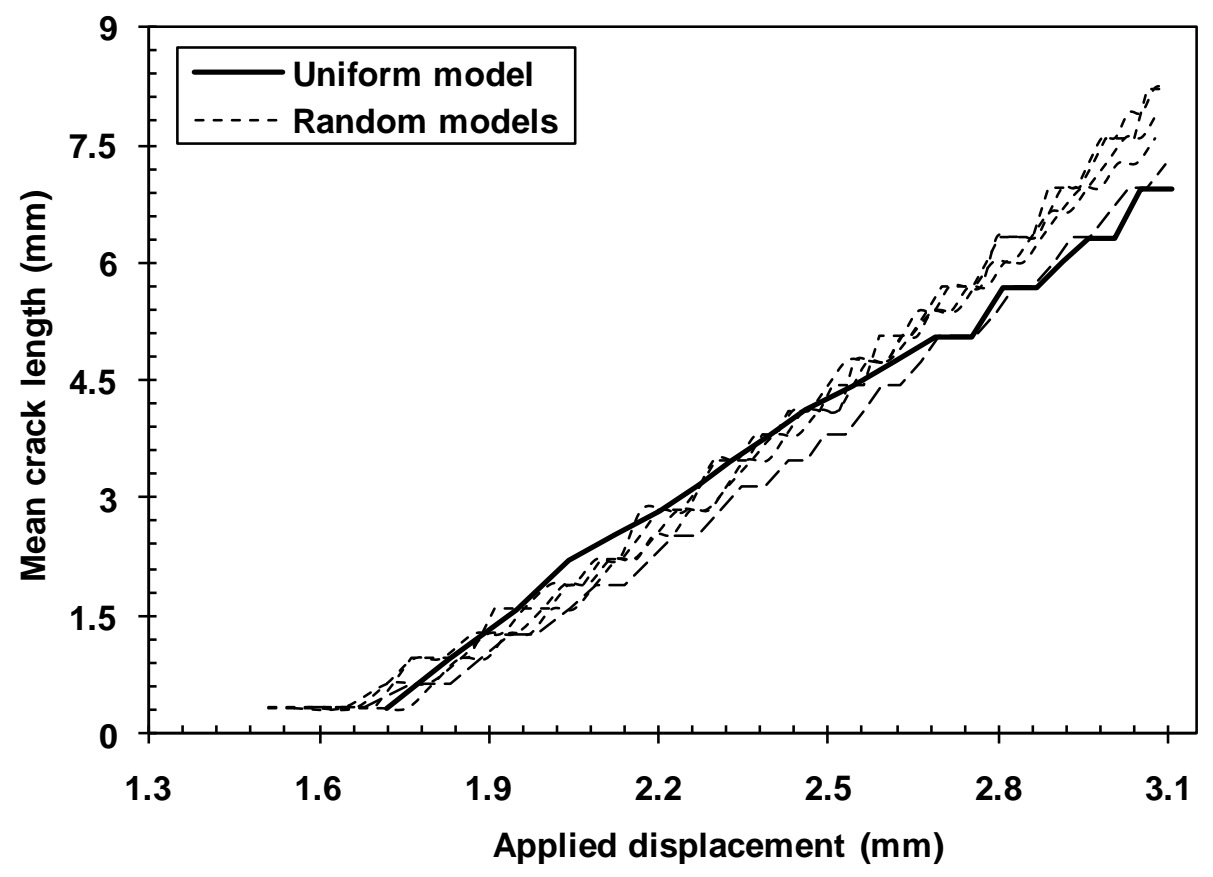

Fig. 14 Mean crack length as a function of applied displacement

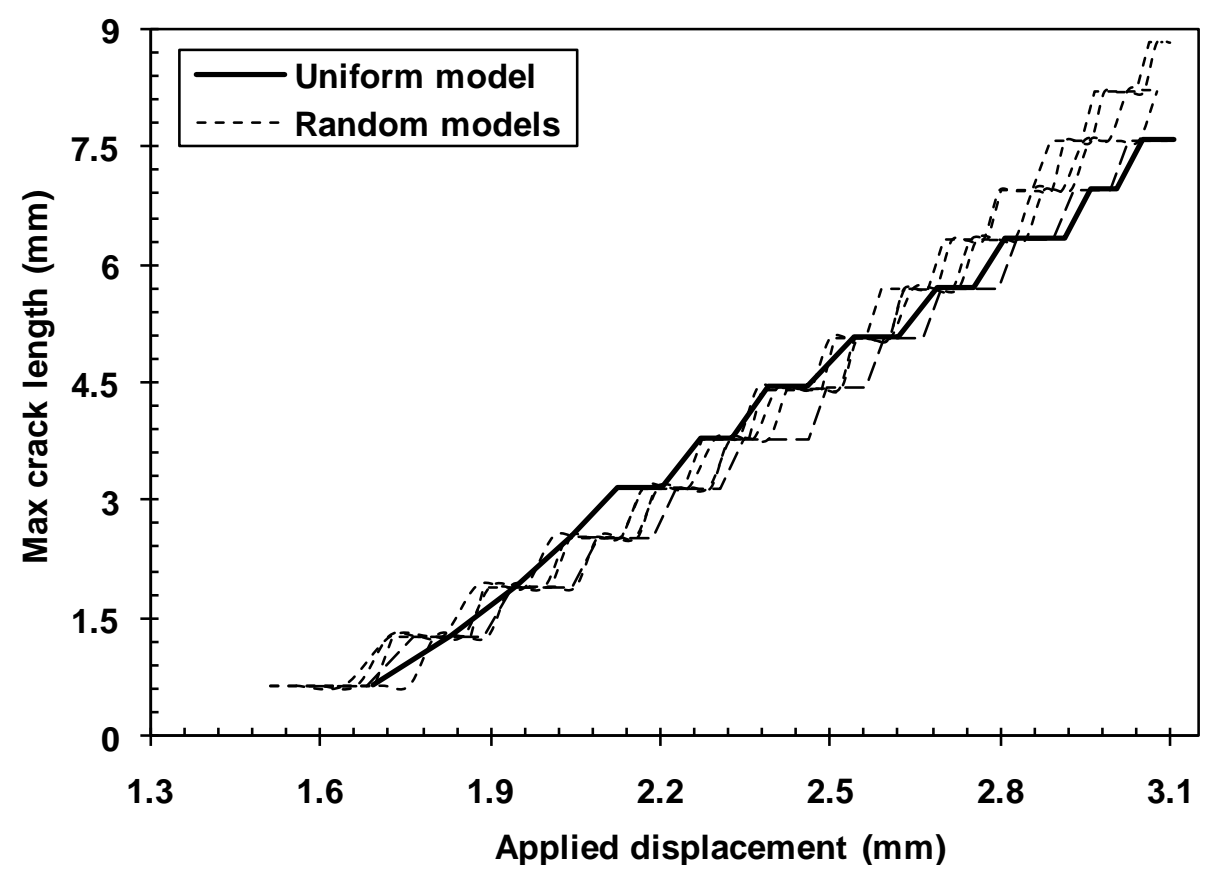

Fig. 15 Max crack length as a function of applied displacement

It was noticed in Fig. 13, Fig. 14 and Fig. 15 that at higher values of displacement, the value of crack length in the random models was always found to be higher than the uniform model. When comparing only the random realizations, the scatter within those was around $20-25 \%$ for the crack lengths. Statistical analyses of the values of crack length for the same level of high applied displacement $(4 \mathrm{~mm})$ were analyzed for various random statistical realizations. The values taken were for the Min length shown in Fig. 12, i.e. the row of elements that occupied 
the full width of the specimen. The probability distribution function based on the twoparameter Weibull's probability distribution function for the crack length values is presented in Fig. 16 (a). This function is negatively-skewed as the mean (13.4) and median (13.1) lie on the left of the mode. The mass of the distribution is concentrated towards the right, which raises concerns, as a higher percentage of the values of crack length lies in this area far away from the value obtained for uniform model. The cumulative distribution function (CDF) is shown in Fig. 16 (b). The descriptive statistics of the distribution are presented in Table 3.

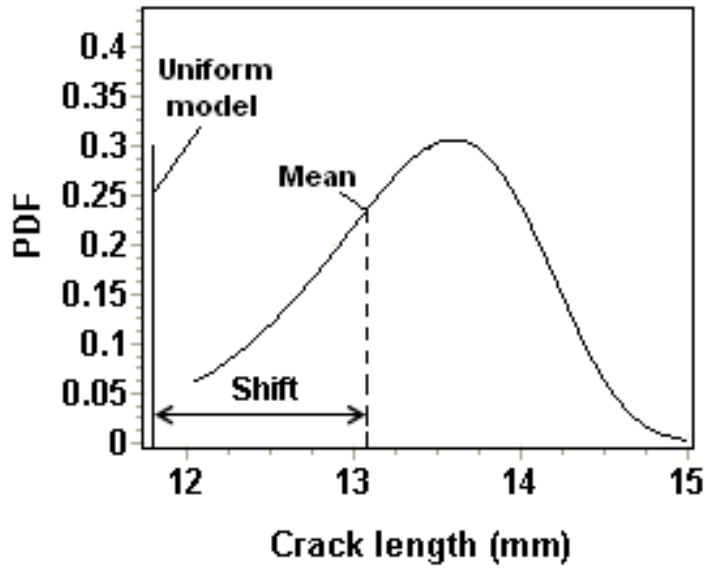

(a)

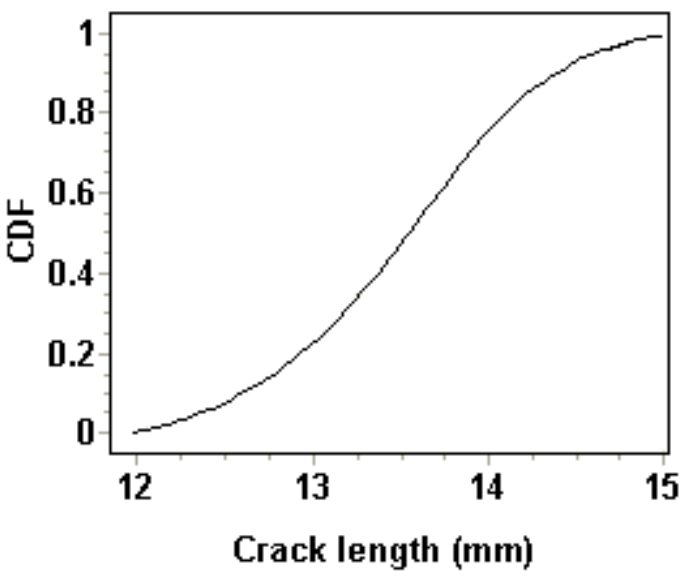

(b)

Fig. 16 Probability density function (a) and cumulative distribution function (b) for "Min" crack length in a DCB ( $\alpha=21.8$ and $\beta=13.6)$ with applied displacement of $4 \mathrm{~mm}$.

Table 3 Descriptive statistics for PDF for "Min" crack length

\begin{tabular}{|l|c|c|c|c|c|c|c|}
\hline Percentile & Min & $10 \%$ & $\begin{array}{c}25 \% \\
(\mathrm{Q} 1)\end{array}$ & $\begin{array}{c}50 \% \\
\text { (Median) }\end{array}$ & $\begin{array}{c}75 \% \\
(\mathrm{Q} 3)\end{array}$ & $90 \%$ & Max \\
\hline Value & 11.9 & 12.5 & 13.1 & 13.1 & 13.8 & 14.4 & 15 \\
\hline
\end{tabular}

\subsection{Analysis of damage area and its variation across width of DCB}

In this section, the damage induced in the DCB specimen is analyzed for various random statistical realizations and a comparison is made with the uniform case. Also presented is the variation of damage across the width of the specimen. To study the variation in damage with displacement, the damage was quantified as the area of failed cohesive elements. The variation in the damaged area (normalized by its maximum magnitude obtained from one of 
the random models), as a function of normalized load is plotted in Fig. 17. The initiation point of each curve corresponds to the critical point, i.e. the moment when the specimen sustained the maximum load on the load-displacement plot. So these curves depict the unstable phase of delamination crack propagation with the decreasing load accompanying an increase in the prescribed displacement. The variations in a number of different parameters, such as the damaged area corresponding to the critical point and the maximum damaged area are readily noticeable in this plot. Comparing the uniform model case with the random models, it is observed that the uniform model showed a significantly higher load-bearing capacity than all the random realizations. Quantitatively, that value was about $12 \%$ higher than the lowest value of critical load in one of the random models. A maximum spread of $20 \%$ was obtained when comparing the values for the maximum damaged area for the uniform model with various statistical realizations. A comparative analysis of only random realizations returned a maximum scatter of around $7 \%$ in the maximum value of the damaged area for the same value of prescribed displacement. A maximum scatter of $10 \%$ in the values of critical load was observed.

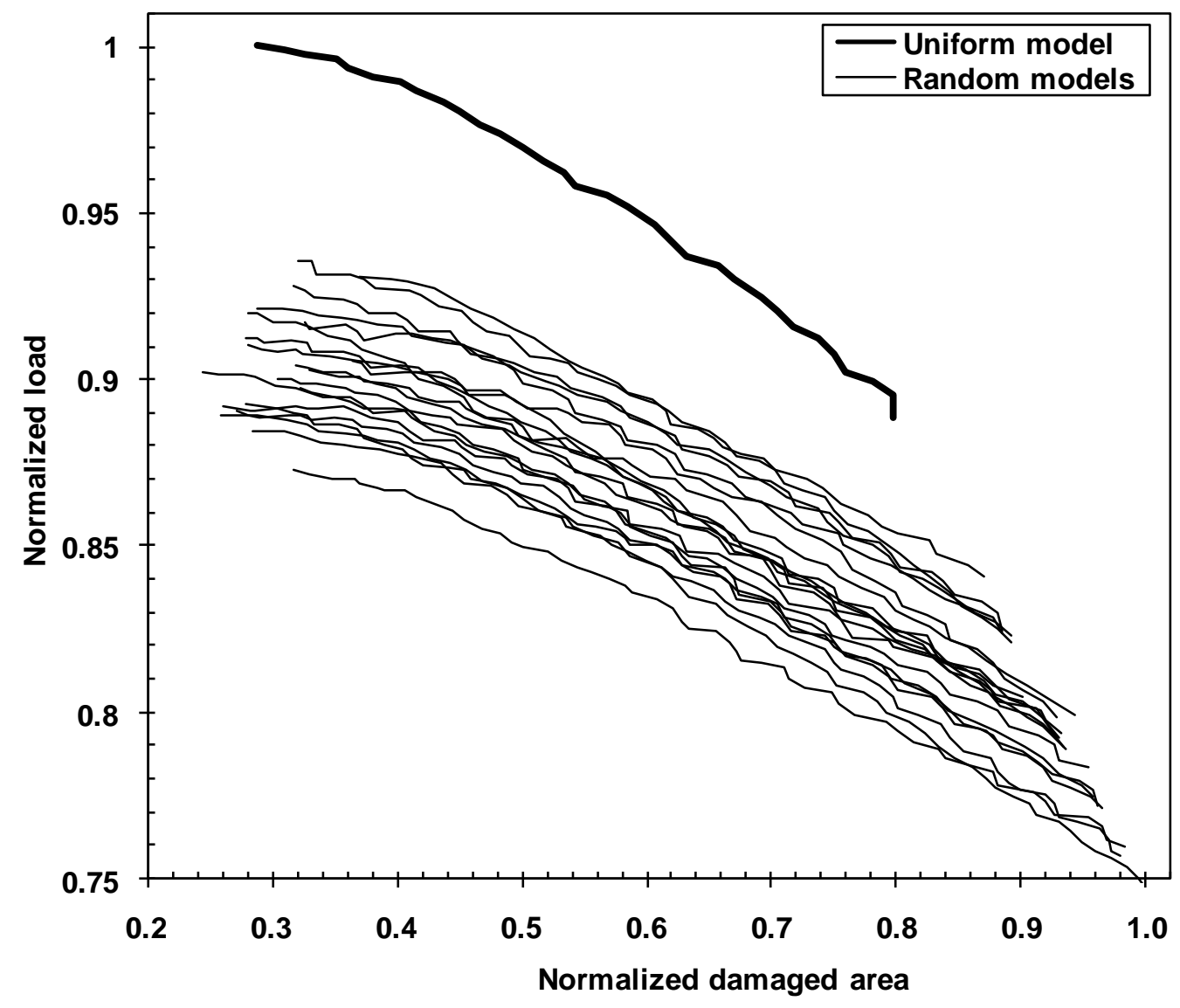

Fig. 17 Load as a function of damage propagation 
Next the variation of damage across the width of the DCB was analysed, as shown in Fig. 18. It can be observed from the figure that the damage variation path for the uniform case (solid line) is a smooth concave curve symmetric about the beam's centre-line of width while the dashed lines represent damage variations for some of the random cases (statistical realizations). In the uniform case, damage initiated from the middle of the width and then propagated towards the edges. A non-smooth fluctuating behaviour was obtained with the random models due to the scatter of the fracture properties within the cohesive layer. These variations were also non-symmetric about the centre-line. Some regions of the cohesive layer, due to a lower value of fracture energy, experienced a fast growth of damage compared to others, as can be seen in Fig. 18.

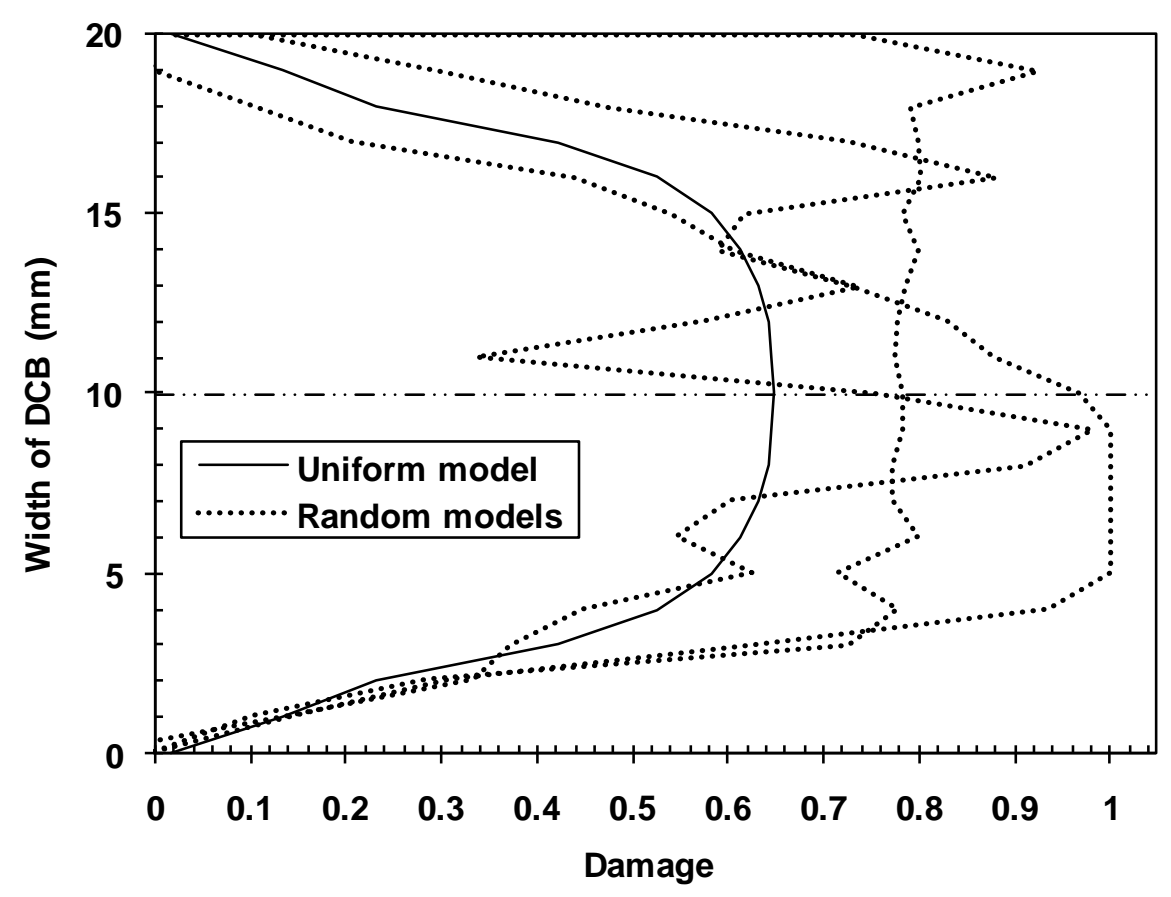

Fig. 18 Variation of damage across DCB width (applied displacement: $2.5 \mathrm{~mm}$ )

\subsection{Analysis of critical loads and displacements}

From the comparison of the uniform model case and the random models in the previous sections, we saw that the uniform model showed a higher load-bearing capacity than all the random realizations. The output statistics for the critical loads and displacements will be presented in this section for the various random statistical realizations analyzed. The probability plot for the critical load is shown in Fig. 19. The plot demonstrates that the values of critical load vary between $73 \mathrm{~N}$ and $78.5 \mathrm{~N}$. 


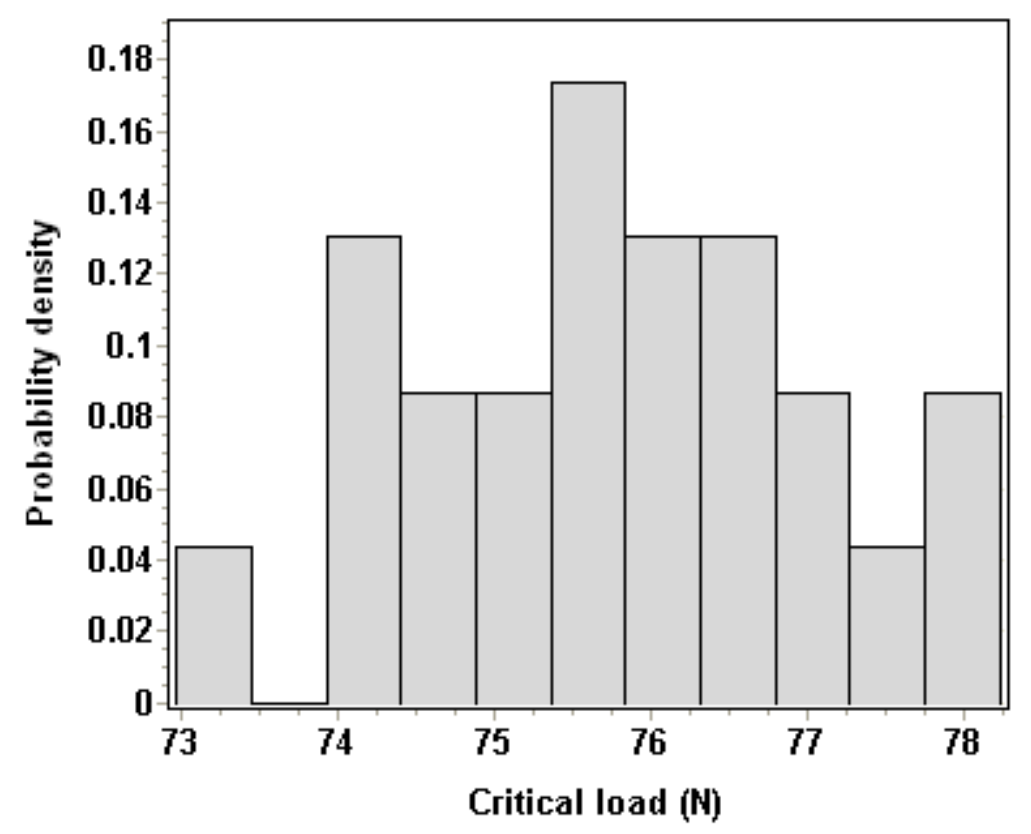

Fig. 19 Probability density plot for critical load

The probability density function for the values of critical load obtained from the analysis of various statistical realizations is shown in Fig. 20. The value obtained from the uniform model $(83.6 \mathrm{~N})$ and the median of the PDF are indicated on this figure. It can be seen that the introduction of stochasticity has the effect of shifting the median (50th percentile) downward, i.e. reducing the critical load compared to uniform model. In the probability density function for random statistical realizations, the distribution function is negatively-skewed (skewed left), depicting a sharply declining tail to the left. This tail end of this distribution function presents values that pose a concern, as these values show the maximum difference compared to the uniform model. The cumulative distribution function (CDF) and descriptive statistics of the distribution are presented in Fig. 21 and Table 4, respectively. The output statistics for the displacement corresponding to the critical load are plotted in Fig. 22. Similar behaviour was obtained for the critical displacement, as seen with the critical load; however, in this case approximately $5 \%$ of the values obtained from the random models were higher than the value for the uniform model. The descriptive statistics of the distribution for output data of critical displacements is given in Table 5. 


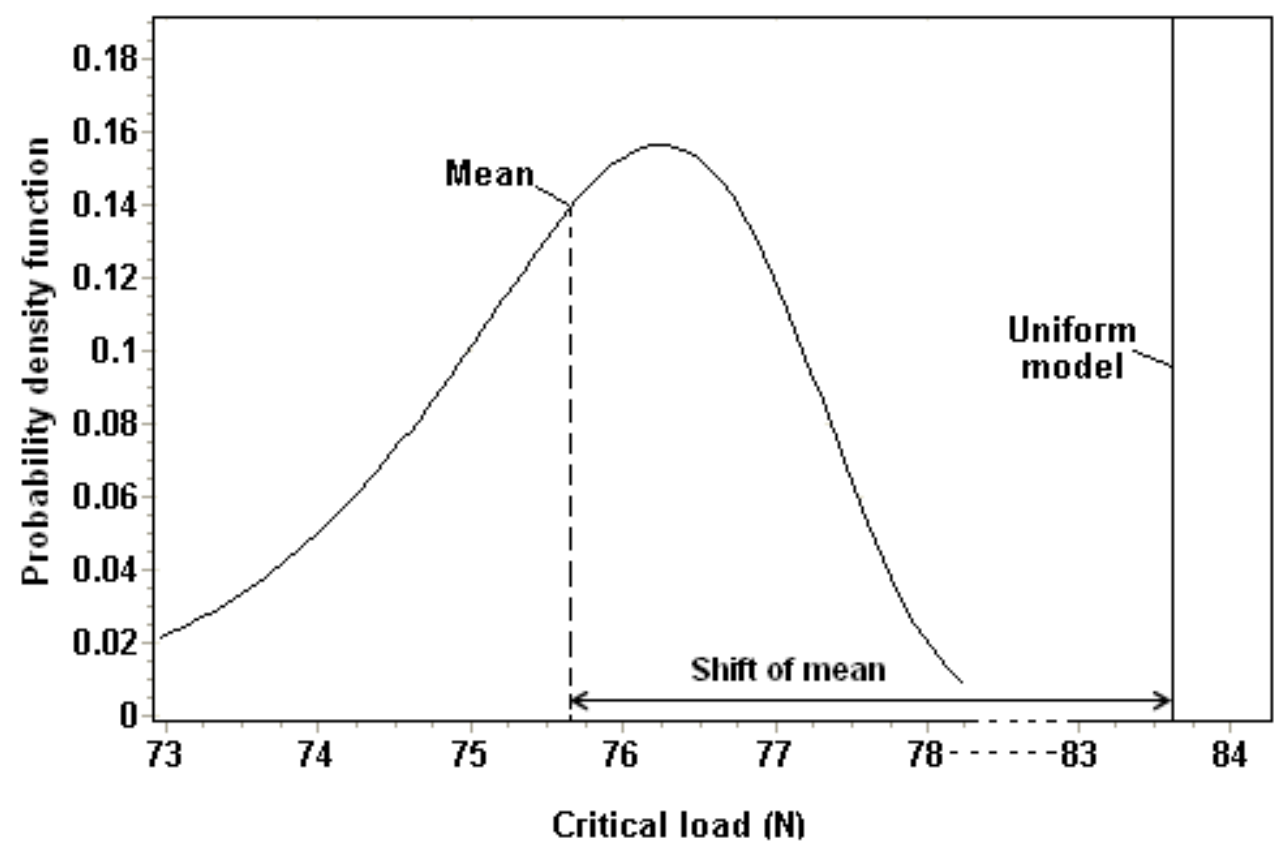

Fig. 20 Probability density function for critical load in a DCB ( $\alpha=67.7$ and $\beta=76.3$ )

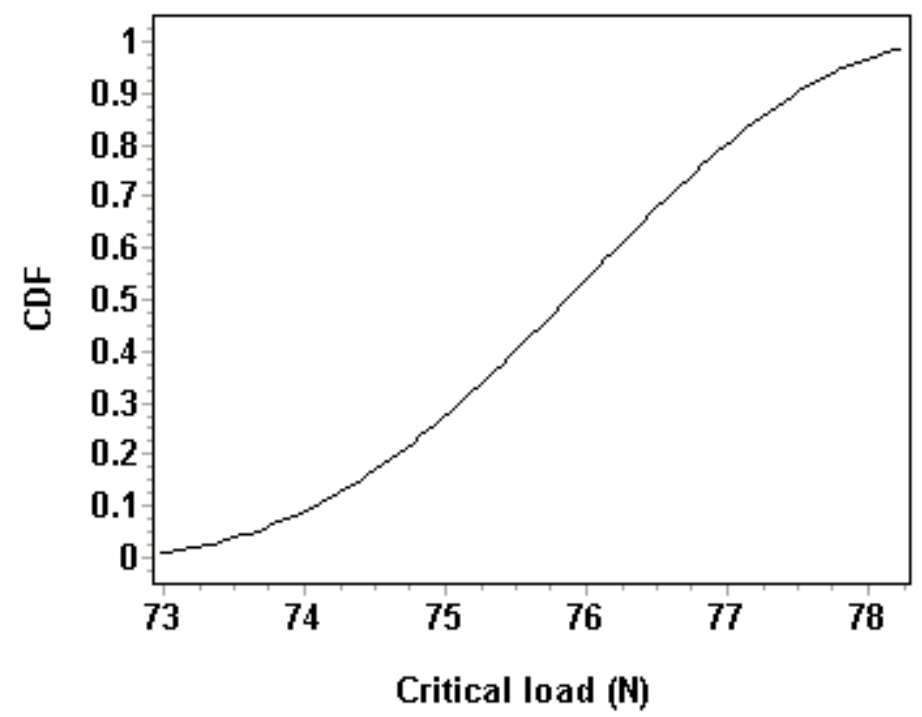

Fig. 21 Cumulative distribution function for critical load in a DCB

Table 4 Descriptive statistics for critical load for various statistical realizations

\begin{tabular}{|l|c|c|c|c|c|c|c|}
\hline Percentile & Min & $10 \%$ & $\begin{array}{c}25 \% \\
\text { (Q1) }\end{array}$ & $\begin{array}{c}50 \% \\
\text { (Median) }\end{array}$ & $\begin{array}{c}75 \% \\
(\mathrm{Q} 3)\end{array}$ & $90 \%$ & Max \\
\hline Value & 73 & 74.1 & 74.7 & 75.7 & 76.7 & 77.7 & 78.2 \\
\hline
\end{tabular}




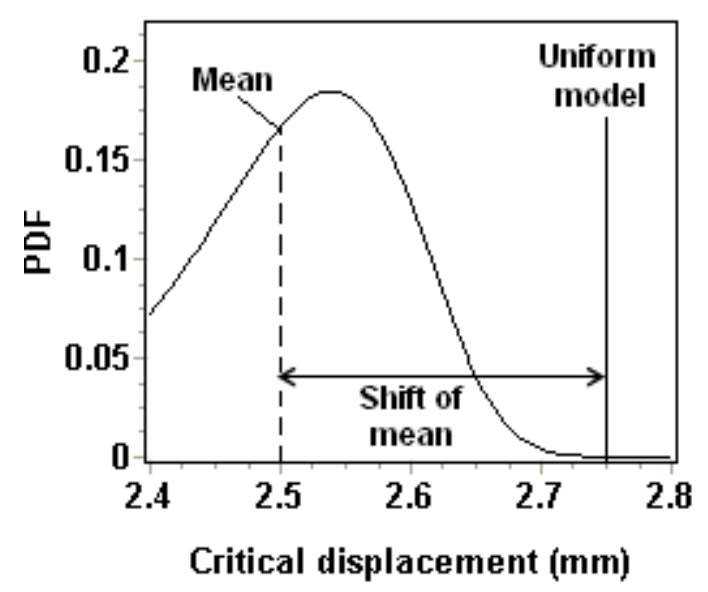

(a)

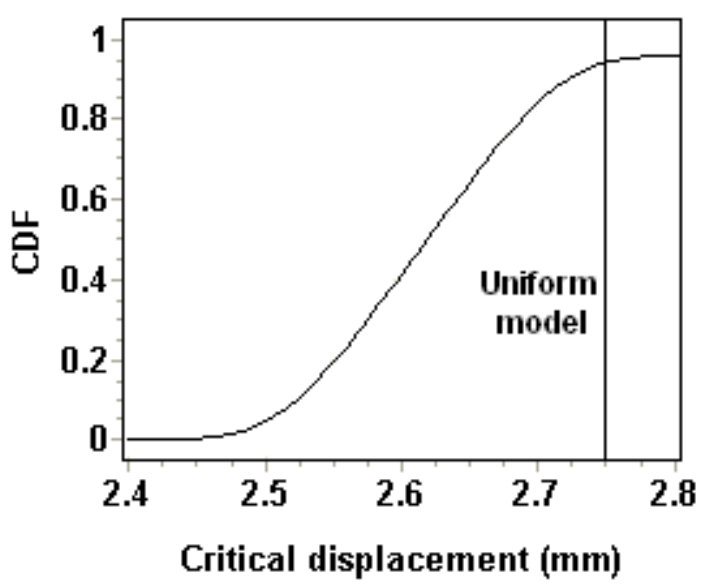

(b)

Fig. 22 Probability density function (a) and cumulative distribution function (b) for critical displacement in a DCB $(\alpha=31.9, \beta=2.54)$

Table 5 Descriptive statistics for critical displacement

\begin{tabular}{|l|c|c|c|c|c|c|c|}
\hline Percentile & Min & $10 \%$ & $\begin{array}{c}25 \% \\
\text { (Q1) }\end{array}$ & $\begin{array}{c}50 \% \\
\text { (Median) }\end{array}$ & $\begin{array}{c}75 \% \\
\text { (Q3) }\end{array}$ & $90 \%$ & Max \\
\hline Value & 2.4 & 2.4 & 2.44 & 2.5 & 2.59 & 2.63 & 2.8 \\
\hline
\end{tabular}

\section{Conclusions}

The underlying objective of this study was to model the delamination failure in CFRP laminated composites in order to highlight the effect of microstructural randomness. One of the models was based on the assumption of uniform microstructure, in which the material properties were constant along the entire cohesive layer. The other model was simulated by directly incorporating the effect of material randomness in which a variation/scatter of cohesive properties (fracture energy and tripping traction) was introduced. More detailed analyses were performed based on three-dimensional models and a number of statistical realizations based on a half-scatter of $50 \%$ of fracture energy were presented. In contrast to the two-dimensional analyses reported previously [8], the results here showed lower load-bearing capacities for most of the random models than that in the uniform model. Also, the damaged area and the crack lengths also showed higher values in the random realizations compared to the uniform case above a certain value of applied displacement. The initiation points for delamination 
cracks also exhibited a considerable uncertainty; delamination initiated at the edges of the specimen and approached the centre-line (across the width) in a uniform model while for random statistical realizations various initiation locations/scenarios were observed. The stress, stress $\sigma_{22}$, also showed random variations ahead of the crack front.

The computational investigation of the effects of material randomness presented demonstrates the need to consider the spatial non-uniformity of properties when analysing damage scenarios and load-carrying capacities in CFRP laminates. Also three-dimensional models need to be employed since in two-dimensional models the variations in fracture properties is only introduced in single dimension, losing the significant effects of variations in properties and subsequent behaviour across the crack/damage front.

In summary, the material randomness in CFRPs can induce randomness in localised damage and this can affect the global properties of laminates and critical failure parameters. These effects can be effectively investigated computationally through the use of stochastic cohesivezone elements. 


\section{References}

[1] Baxevanakis, Jeulin, C.D., Renard, J.: Fracture statistics of a unidirectional composite. Int. J. Fracture 73, 149-181 (1995)

[2] Silberschmidt, V.V.: Scaling and multifractal character of matrix cracking in carbon fibre-reinforced cross-ply laminates. J. Mech. Compos. Mater. Structures 2, 243-255 (1995)

[3] Silberschmidt, V.V.: Matrix cracking in cross-ply laminates: effect of randomness. Compos. Part A: Appl. Sci. Manuf. 36, 129-135 (2005)

[4] Trias, D., Costa, J., Mayugo, J.A., Hurtado, J.E.: Random models versus periodic models for fibre-reinforced composites. Comput. Mater. Sci. 38, 316-324 (2006)

[5] Silberschmidt, V.V.: Effect of micro-randomness on macroscopic properties and fracture of laminates. J. Mater. Sci. 41, 6768-6776 (2006)

[6] Yanga, Z.J., Su, X.T., Chen, J.F., Liu, G.H.: Monte Carlo simulation of complex cohesive fracture in random heterogeneous quasi-brittle materials. Int. J. Solids Structures 46, 3222-3234 (2009)

[7] Khokhar, Z.R., Ashcroft, I.A., Silberschmidt, V.V.: Development of delamination in cross-ply laminates: effect of microstructure. Key Engng Mater. 413-414, 229-236 (2009)

[8] Khokhar, Z.R., Ashcroft, I.A., Silberschmidt, V.V.: Simulations of delamination in CFRP laminates: Effect of microstructural randomness. Comput. Mater. Sci. 46, 607613 (2009)

[9] Li, S.: General unit cells for micromechanical analyses of unidirectional composites. Compos. Part A: Appl. Sci. Manuf. 32, 815-826 (2001)

[10] Ashcroft, I.A., Khokhar, Z.R., Silberschmidt, V.V.: Modelling the effect of microstructural randomness on the mechanical response of composite laminates through the application of stochastic cohesive zone elements. Comput. Mater. Sci. 52, 95-100 (2011)

[11] Yang, Z., Xu, X.F.: A heterogeneous cohesive model for quasi-brittle materials considering spatially varying random fracture properties. Computer Meth. Appl. Mech. Engng 197, 4027-4039 (2008) 